\title{
Effects of Pristane on Cytochrome P450 Isozyme Expression in Rat Tissues
}

\author{
Carolyn B. Howard ${ }^{1 *}$, Jacqueline Samuel ${ }^{1}$, Shalonda B. Henderson ${ }^{1}$, Jacqueline Stevens ${ }^{1}$, Paul E. Thomas ${ }^{2}$ and \\ Marvin A. Cuchens ${ }^{3}$ \\ ${ }^{1}$ Breast Cancer Research Laboratory, Department of Biology, and NIH-Center for Environmental Health, College of Science, \\ Engineering and Technology, Jackson State University, 1400 Lynch Street, P. O. Box 18540, Jackson, Mississippi, USA. \\ ${ }^{2}$ Laboratory for Cancer Research, College of Pharmacy, Rutgers University, Piscataway, NJ 08855-0789, U S A. \\ ${ }^{3}$ Department of Microbiology, University of Mississippi Medical Center, Jackson, MS 39216-4505, USA. \\ ${ }^{*}$ Correspondence to Dr. Carolyn B. Howard. Email: carolyn.b.howard@jsums.edu
}

Received: 15 November 2004 / Accepted: 06 February 2005 / Published: 30 April 2005

\begin{abstract}
Chemical carcinogenesis studies are powerful tools to obtain information on potential mechanisms of chemical factors for malignancies. In this study Western blot analyses, using monoclonal antibodies specific for three different cytochrome P450 (CYP) isozymes (CYP1A1, CYP1A2 and CYP2B), were employed to examine the effect(s) of 3-methylcholanthrene and/or pristane (2,6,10,14-tetramethylpentadecane) on the basal and inducible levels of expression of CYP proteins within Copenhagen rat tissues. Pristane exposure led to tissue specific differences in the CYP isozymes expressed and elicited increased CYP protein expression over 3methylcholanthrene induced levels in microsomes isolated from liver, Peyer's Patches, and thymus. Within the context of the chemical carcinogenesis model employed in this study, these observations correlated with the induction of B-cell malignancies by low doses of 3-methylcholanthrene and of thymic lymphomas by a high 3methylcholanthrene dose. The data suggest that pristane treatment affects CYP isozyme expression. This pristane-mediated effect clearly could be a contributing factor in the chemical carcinogenesis of the previously observed lymphoid malignancies, and a possible basis for the tumor enhancing effects of pristane.
\end{abstract}

Keywords: Pristane; Cytochrome P450 (CYP); monoclonal antibodies; 3-methylcholanthrene; phenobarbital; Western blots

\section{Introduction}

Pristane is a ubiquitous isoprenoid which has been shown to induce B-cell plasmacytoma, following intraperitoneal administration into genetically susceptible BALB/cAnPt mice [1-6]. Pristane treatment altered CYP1A activity and polyamine regulation [7], and has been shown to affect several biological responses including changes in membrane fluidity, chromatin conformation and histone protein expression [8]. In addition, pristane exhibited tumor enhancing properties in our Copenhagen rat model in which direct Peyer's patches (PP) injection with a low dose of 3methylcholanthrene (3-MC) led to only B lymphoid malignancies, a high 3-MC dose treatment induced thymic lymphomas but no B cell malignancies, and cotreatment with 3-MC and pristane lead to higher frequency and decreased latency of 3-MC induced lymphoid malignancies [9]. Also, pristane elicited marked effects on transactivation of transfected genes
[10-13]. These activities are comparable to 12-Otetradecanoylphorbol 13-acetate (TPA), a known tumor promoter [14]. However, based on the disparity of the results obtained with TPA and pristane in protein kinase $\mathrm{C}$ substrate phosphorylation and activation of the chloramphenicol acetyltransferase genes [10] it appears that TPA and pristane exhibit different tumor promotion mechanism(s) of action in chemical carcinogenesis. TPA induces CYP1A and simultaneously suppresses CYP2B [15], but the mechanism of CYP1A induction by TPA appears to differ from other TPA inducible functions, as well as from those elicited by 3-MC [1113]. TPA intercalates into DNA and modifies histone phosphorylation, thus altering the super-structure of chromatin [14]. On the other hand, pristane is absorbed from the gut contents, disseminates through the body, and the majority of pristane is localized to the plasma membranes [16].

Membrane perturbation by pristane possibly leads to alterations in regulation of many different cellular 
products including cytochrome P450 (P450) isoforms, which are also membrane associated. Using pristane and the carcinogen 3-MC [17], which induces P450 isozymes (products of the CYP genes), we examined the effect(s) of pristane on CYP isozyme expression within the suspected target organs. Not only are CYP isozymes specifically regulated [18], they are inducible [19-30] and catalyze the biotransformation of both exogenous and endogenous compounds to highly reactive intermediates which can cause toxicity and/or carcinogenicity [25-30]. We undertook these studies in light of the fact that pristane influences CYP1A activity in a 3-methylcholanthrene (3-MC) dose dependent manner [7], leading to speculation as to whether or not tissue specific expression of CYP protein correlated with CYP1A activity in the preferential induction of lymphoid malignancies.

\section{Materials and Methods}

\section{Chemicals and Reagents}

The following commercially available chemicals were employed in this study: 3-methylcholanthrene (3-MC) and $\beta$ mercaptoethanol from Eastman Kodak Company (Rochester, NY); 2,6,10,14-tetramethylpentadecane (pristane), sunflower seed oil, bovine serum albumin, sodium dodecyl sulfate (SDS), ammonium persulfate, N,N,N',N'tetramethylethylenediamine (TEMED), nitro blue tetrazolium (NBT) tablets, 5-bromo-4-chloro-3-indolyl phosphate (BCIP), trizma base, Coomassie blue R250, bromphenol blue, $\alpha$-nitrocellulose membrane, acrylamide, bisacrylamide, glycine, glycerol, goat serum from Sigma Chemical Company (St. Louis, MO); protein assay reagent from Biorad Laboratories (Richmond, CA); and sodium phenobarbital (PB) from J.T. Baker Chemical Company (Phillipsburg, NJ). All other inorganic salts and solvents used were of analytical grade. Alkaline phosphatase conjugated and horseradish peroxidases labelled secondary antibodies were obtained from Southern Biotechnology Assoc., Inc. (Birmingham, AL). Monoclonal antibodies (MAbs) specific for CYP1A1, CYP1A2, and CYP2B isozymes have been reported [31-32].

\section{Treatment of Animals}

Female Copenhagen rats (8-10 weeks old) were initially obtained from the National Cancer Institute, Frederick, MD., and were subsequentially bred and maintained under NIH guidelines. Animals were provided with a commercial diet (Harlan Teklan Laboratory, Madison, WI.), and water ad libitum. All rats were anesthetized prior to injections, surgery or euthanasic procedures. The Copenhagen rat strain was selected due to its low rate of spontaneous tumor development [33]. Where indicated, pristane was administered as a $1 \mathrm{ml}$, intraperitoneal (i.p.) injection either alone, 2 weeks prior, or 4 weeks prior to either animal sacrifice or 3-MC treatment (See Table 1). Age matched rats were separated into specified groups containing six animals per group. Group 1 (BASAL) rats were normal, untreated controls $\left(\mathrm{H}_{2} \mathrm{O}\right.$ or SSO treated rats served as controls as well, and yielded similar results). Group 2 rats were treated with only pristane and were sacrificed either two weeks or four weeks after pristane priming. Group 3 rats were treated with only 3-MC (3, 12 or 24 hrs; directly injected into Peyer's patches (PP) with $0.05 \mu \mathrm{g}, 5 \mu \mathrm{g}$, or $500 \mu \mathrm{g}$ of $3-\mathrm{MC}$ ) as previously described [9]. The 3-MC, dissolved in sunflower seed oil (SSO), was injected into the five PP which were most distal on the small intestine. Within group 4, rats received 3-MC and pristane (2 wks) co-treatment.

Data obtained from Western analyses of tissue samples (group 3-4 rats) employed 3-MC doses which were carcinogenic in our rat tumor model. However, these treatment protocols generated expression levels of CYP which were at the lower limits of detection in PP and thymus (THY). Therefore, in order to further examine the effects of pristane on CYP isozyme expression in PP and THY, protocols using very high doses of inducers were employed. To maximally induce CYP1A1 and CYP1A2 [19, 24], 3-MC (25mg/kg/day for 4 consecutive days; $\mathrm{M} \mathrm{VH}$ ) dissolved in SSO was administered i.p. within group 5 rats. Phenobarbital (PB), used as a control, $(75 \mathrm{mg} / \mathrm{kg} /$ day for 4 consecutive days) was dissolved in $\mathrm{H}_{2} \mathrm{O}$ and injected i.p. into group 6 rats, as an efficient means to induce CYP2B1 and 2B2 $[30,32]$. It should be noted that animals were injected and sacrificed in the morning whenever possible in order to minimize possible variations from circadian rhythms. All animals were sacrificed one day after the last dose of pristane, 3-MC, PB or control vehicle treatment.

\section{Preparation of Microsomes}

Microsomal fractions were prepared from LIV, PP, and THY, of rats according to a previously described method $[7,34]$. As indicated, tissues were initially rinsed in ice cold $1.15 \% \mathrm{KCl}$ and homogenized in four volumes of a buffer containing $0.1 \mathrm{M} \mathrm{KCl}, 0.1 \mathrm{M}$ Tris-acetate, and $1 \mathrm{mM}$ EDTA (pH 7.4), using a Tekmar Tissumizer and sonified at $50 \%$ power for 4 consecutive $15 \mathrm{sec}$ bursts. Homogenates were centrifuged at $10,000 \times \mathrm{g}$ for $20 \mathrm{~min}$ at $4^{\circ} \mathrm{C}$. The supernatants were removed and centrifuged at 100,000 x g for $1 \mathrm{hr}$ at $4^{\circ} \mathrm{C}$. The microsomal pellets were then suspended in a buffer containing $0.1 \mathrm{M} \mathrm{Na}_{2} \mathrm{H}_{2} \mathrm{P}_{2} \mathrm{O}_{7}$ and 1 $\mathrm{mM}$ EDTA (pH 7.4), and frozen at $-80^{\circ} \mathrm{C}$ until used for Western blot analyses.

Rat microsomes from each of the treatment groups (group 1 - group 6) were prepared and analyzed by Western blots. Each microsomal preparation and each Western blot analysis was repeated in four different experiments in order to statistically compare and evaluate each band such that even marginal changes in band intensity represented effects of the inducer.

\section{Western Blot Analyses}

For determinations of levels of CYP protein expression, samples were prepared in Laemmli buffer (Invitrogen) consisting of $125 \mathrm{mM}$ Tris- $\mathrm{HCl}(\mathrm{pH} 6.8), 10$ $\mathrm{mM}$ DTT, $17.4 \%$ glycerol, $3 \%$ SDS, $0.025 \%$ bromophenol blue, and $10 \mathrm{mM} \quad \beta$-mercaptoethanol. Samples were loaded into wells at the indicated concentration of microsomal protein (a concentration of $20 \mu \mathrm{g}$ of microsomal protein/track, with the exceptions of liver microsomes from very high dose $3-\mathrm{MC}$ and $\mathrm{PB}$ 
treated rats which were loaded at $0.2-2 \mu \mathrm{g} / \mathrm{track}$ and thymic samples which were loaded at $200 \mu \mathrm{g} /$ track). Total protein concentrations were determined by the methods of Bradford and Lowry, using the Bio-Rad Protein Assay reagents. Based on chemical assays for protein (Biorad) and densitometric scans of Coomassie blue stained gels (see below), the total amount of protein loaded onto each gel lane was within $10 \%$ of the average load per well.

SDS-gel electrophoresis was performed according to Laemmli using $16 \mathrm{~cm}$ long gels with a $12 \%$ resolving gel with a $4 \%$ stacking gel for the resolution of microsomal proteins. For each experiment, there were four identical gels run overnight, of which three were electrophoretically transferred to nitrocellulose and used for Western blot analyses, and one gel was used for staining with Coomassie blue dye. The nitrocellulose filters were probed with MAbs specific for CYP1A1 or CYP1A2 (3-MC inducible isozymes), as well as CYP2B (PB inducible CYP isozymes), including CYP2B1 and CYP2B2 which share 96\% sequence homology [32], as previously described, followed by alkaline phosphatase conjugated second antibody probing and development with NBT and BCIP. Molecular weight markers were included on each gel in order to identify the expected area of migration of the CYPs (CYP1A1, 55 or $56,000 \mathrm{kDa}$; CYP1A2, 52,000 kDa; and CYP2B1 and 2B2, 51,000 kDa and $52,000 \mathrm{kDa}$, respectively) under these conditions.

Electrophoresed proteins were transferred to nitrocellulose filter paper by electroblotting overnight at $15 \mathrm{~V}$ in pre-chilled transfer buffer containing $25 \mathrm{mM}$ Tris, $\mathrm{pH} 8.3,192 \mathrm{mM}$ glycine, and 20\% methanol. Filters were blocked with non-fat dry milk or bovine serum albumin, dependent upon recommended conditions for each monoclonal antibody. Following rinsing, membranes were probed with primary antibody, washed, incubated with HRP-conjugated secondary antibody and developed by enhanced chemiluminenscence.

\section{Immunoquantification}

Relative densities $\left(\mathrm{OD} / \mathrm{mm}^{2}\right)$ of Coomassie stained gels or developed immunoblots were quantified with a densitometer interfaced with a computer equipped with the ImageQuant Software package (Molecular Dynamics Inc., Sunnyvale, CA). The densitometer generated a general graphics file which was transferred to Microsoft Excel graphics programs for Windows (Microsoft Corp., Redmond, WA) and analyzed using volume integration parameters $\left(1\right.$ pixel $=.007744 \mathrm{~mm}^{2}$ ).

\section{Results}

Western Blot Analyses of LIV, PP and THY Tissues following Various Treatment Protocols

Within hepatic samples from untreated rats (Group 1), no CYP1A1 was detected, although basal levels of CYP1A2 and CYP2B proteins (note two bands since our $M A b$ probe specific for $C Y P 2 B$ detects both the CYP2B1 and the CYP2B2 isozymes) were apparent. However, pristane treatment (Group 2) affected the expression of the latter mentioned CYPs without affecting CYP1A1 (see Fig. 1). This effect was best demonstrated with $20 \mu \mathrm{g}$ and $2 \mu \mathrm{g}$ of microsomal protein for CYP1A2 and CYP2B, respectively. Based on the densitometric scans of these profiles pristane elicited 1.7 and 1.4 fold increases in CYP1A2 and 1.6 and 1.5 fold increases in CYP2B after 2 and 4 weeks post pristane treatment, respectively.

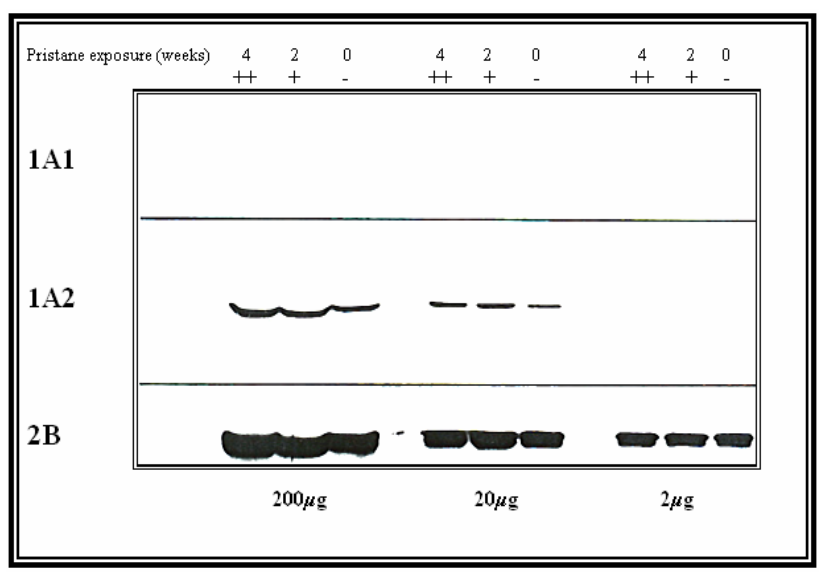

Figure 1: The effect of pristane on basal levels (C) of CYP in LIV. Immunoblots of LIV samples from unprimed (-) or pristane primed $(+=1 \mathrm{ml}$ pristane i.p. 2 wk before animal sacrifice; $++=1 \mathrm{ml}$ pristane i.p. 4 wk before animal sacrifice) rats which did not receive 3-MC were used to establish background levels for untreated LIV. Hepatic microsomes were applied at $2 \mu \mathrm{g}, 20 \mu \mathrm{g}$ or $200 \mu \mathrm{g}$ of microsomal protein/well to determine the optimal loading concentration of LIV microsomal proteins necessary for detection of CYPs using Western blot procedures. Four duplicate gels were run, three were probed with the indicated, specific MAb probes (anti-CYP1A1, CYP1A2 and CYP2B) and one was stained with Coomassie blue R-250 to establish loading consistencies.

Representative data obtained from LIV samples isolated from rats which were either untreated (Group 1), treated with a single i.p. injection of only pristane (Group 2) or treated with a very high dose of either 3$\mathrm{MC}$ or $\mathrm{PB}$ with or without the pristane treatment are presented (Fig. 2). It is important to note that only $0.2 \mu \mathrm{g}$ of microsomal protein per lane was loaded onto the gel used to generate this immunoblot. This was $10 \mathrm{x}$ less than the lowest amount $(2 \mu \mathrm{g})$ depicted in Fig. 1 (i.e. basal levels of CYP1A2 protein were below detection limits within this particular gel since at least $20 \mu \mathrm{g}$ of microsomal protein was needed). However, following very high dose treatment with 3-MC (Group 5), not only were appreciable levels of CYP1A2 detected, but also CYP1A1; the increase in CYP1A2 represented a $>41$ fold increase over basal control levels whereas the amount of CYP1A1 increased at least 1000 fold (assuming $>200 \mu \mathrm{g}$ of microsomal protein was necessary to detect basal levels; see Fig. 1, i.e. no bands with CYP1A1). As expected, neither CYP1A1 nor CYP1A2 were elevated after very high dose PB treatment (Group 6), whereas CYP2B was clearly induced (24 fold greater than basal, based upon densitometric scans). Again, pristane affected the responses, although in a somewhat different fashion. 
Specifically, pristane treatment (2wk or $4 \mathrm{wk}$ ) led to decreased expression of CYP1A1 protein (1.4 and 1.2 fold, respectively), CYP1A2 protein (1.3 and 1.1 fold, respectively) and $\mathrm{CYP} 2 \mathrm{~B}$ protein (2.9 and 1.5 fold, respectively) after very high dose treatment with $3-\mathrm{MC}$, as well as decreases in CYP2B protein (1.3 and 1.2 fold, respectively) after very high dose treatment with $\mathrm{PB}$.

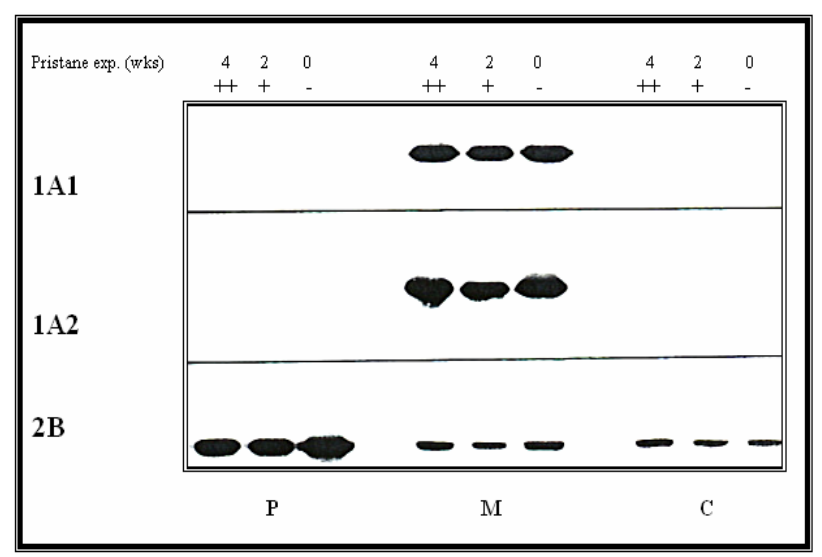

Figure 2: Immunoblot of rat LIV microsomes from untreated $(\mathrm{C})$, very high dose 3-MC treated (3-MC was dissolved in SSO and administered i.p. at $25 \mathrm{mg} / \mathrm{kg} /$ day for 4 consecutive days) or $\mathrm{PB}$ (PB was dissolved in $\mathrm{H}_{2} \mathrm{O}$ and administered i.p. at $75 \mathrm{mg} / \mathrm{kg} /$ day for 4 consecutive days treated animal). All microsomal samples were applied to the gel at a final concentration of $0.2 \mu \mathrm{g}$ of microsomal protein/well to access the effects of 3-MC (M) or PB (P) very high dose treatment on the expression of CYP in unprimed (-) or pristane primed $(+$ $=1 \mathrm{ml}$ pristane i.p. $2 \mathrm{wk}$ before animal sacrifice; $++=1$ $\mathrm{ml}$ pristane i.p. $4 \mathrm{wk}$ before animal sacrifice) LIV samples. This Western blot analyses served as a positive control for very high dose treated LIV. Three of the 4 duplicate gels were antibody probed (CYP1A1, CYP1A2, or CYP2B as indicated) and one was proteinstained as described in the Methods and Materials section.

The effects of carcinogenic doses of 3-MC (within the animal model employed) on hepatic CYPs were also examined. A representative immunoblot depicting the effects of 3-MC, 3 and $12 \mathrm{hr}$ post injection into the PP of either unprimed (Group 3) or pristane primed (Group 4) rats are depicted in Fig. 3. Note that the amount of LIV microsomal protein was 100 fold more than that employed in Fig. 2; hence the basis for detection of CYP1A2. Again, CYP1A1 levels were below detectable levels. Also, as previously observed, pristane treatment led to increased expression over basal levels of CYP2B. However, increased expression over constitutive levels of CYP1A2 in pristane treated rats (without 3-MC exposure) was detected by employing this amount of protein. In addition, increased amounts over untreated control levels of CYP1A2 were observed after 3-MC exposure. Unexpectedly, less CYP1A2 appeared to be present in the LIV $3 \mathrm{hr}$ after PP injection with $500 \mu \mathrm{g}$ than with 0.05 or $5 \mu \mathrm{g}$ of $3-\mathrm{MC}$. Upon co-treatment with pristane, CYP1A2 levels (when compared to hepatic levels at the respective times from rats not treated with pristane): 1) decreased after $3 \mathrm{hr}$ and increased after $12 \mathrm{hr}$ at the $0.05 \mu \mathrm{g} 3-\mathrm{MC}$ treatment, 2) decreased after 3 and $12 \mathrm{hr}$ at the $5 \mu \mathrm{g} 3-\mathrm{MC}$ treatment, and 3) increased after $3 \mathrm{hr}$, but decreased after $12 \mathrm{hr}$ at the $500 \mu \mathrm{g} 3-\mathrm{MC}$ treatment. With respect to CYP2B, pristane co-treatment led to levels which were increased after 3 and $12 \mathrm{hr}$ at the $0.05 \mu \mathrm{g} 3-\mathrm{MC}$ treatment, but decreased after 3 and $12 \mathrm{hr}$ at the $5 \mu \mathrm{g}$ and $500 \mu \mathrm{g} 3-\mathrm{MC}$ treatments.

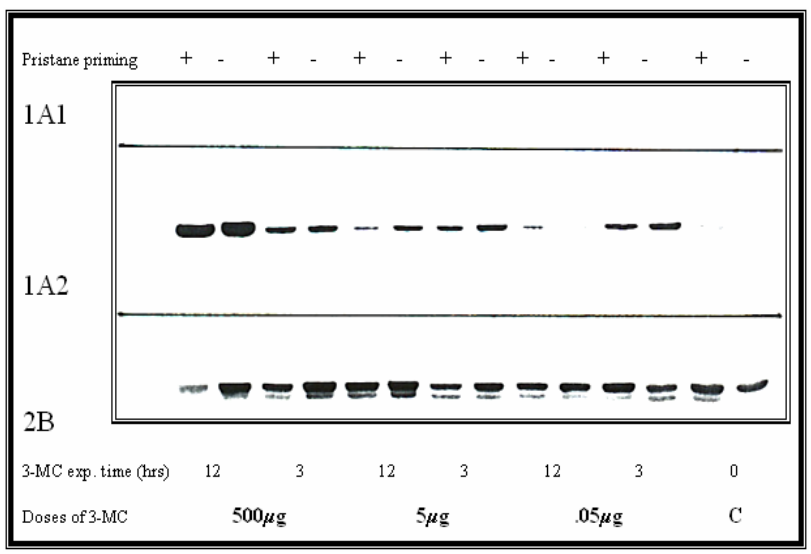

Figure 3: The effects of carcinogenic doses $(0.05,5$ or $500 \mu \mathrm{g})$ of $3-\mathrm{MC}$ and pristane $(-=$ unprimed; $+=$ pristane primed) on CYP levels in the LIV. Animals were treated with indicated doses of 3-MC for 3 or $12 \mathrm{hr}$ exposure times. All microsomal samples were applied to the gel at a final concentration of $20 \mu \mathrm{g}$ of microsomal protein/well. The procedure for Western blot analyses is described in the Methods and Materials section. Three of the 4 duplicate gels were probed with the indicated antibody probe and one was stained with Coomassie blue. Equivalent concentrations of untreated control samples $(\mathrm{C}=$ control liver $)$ were included on the gels as indicated.

PP were also examined with respect to the constitutive expression (Group 1) of the CYPs, as well as CYP protein levels after exposure to pristane (Group 2) and/or 3-MC treatment with very high dose (Group 5) or carcinogenic (Groups 3 and 4) protocols. Neither CYP1A1, CYP1A2, nor CYP2B was detected within the $\mathrm{PP}$ of untreated rats (Group 1), nor were any of these 3 isozymes of CYP detected following very high dose 3MC (Group 5) or PB (Group 6) treatment. Furthermore, treatment with only pristane (Group 2) did not elicit detectable levels of the CYP1A isozymes. However, PP injection with $500 \mu \mathrm{g}$ of 3-MC (Group 3) resulted in increased levels of CYP1A2, but not CYP1A1, without affecting basal levels of CYP2B (see Fig. 4). This was the only dose of 3-MC which elicited appreciable levels of CYP1A2 within PP. Note that the maximum increase in CYP1A2 protein was $12 \mathrm{hr}$ after PP injection with 3$\mathrm{MC}$; a decrease was observed $24 \mathrm{hr}$ post exposure. Cotreatment with pristane (Group 4) elicited increased expression of CYP1A2 3hr post 3-MC injection of the $\mathrm{PP}$, although no apparent differences in the untreated versus pristane treated rats injected with $500 \mu \mathrm{g}$ of $3-\mathrm{MC}$ were subsequently observed in microsomal protein samples prepared either $12 \mathrm{hr}$ or $24 \mathrm{hr}$ post $3-\mathrm{MC}$ treatment. 


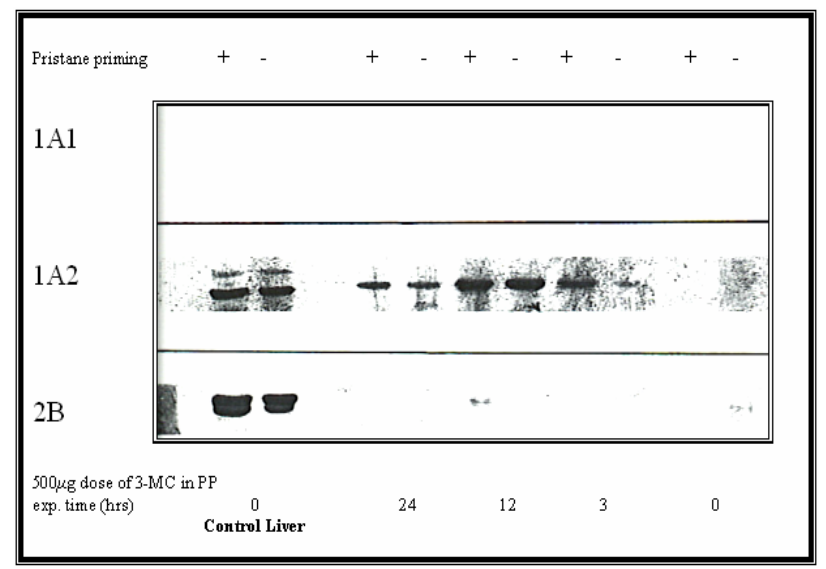

Figure 4: The temporal effects of $500 \mu \mathrm{g}$ of $3-\mathrm{MC}$ injected into the PP in unprimed (-) or pristane primed $(+)$ rats on the expression of CYPs in PP. Microsomal samples were prepared from rat tissues following treatment with $500 \mu \mathrm{g} 3-\mathrm{MC}$ dose for the 3, 12 and $24 \mathrm{hr}$ exposure time with or without pristane priming. Samples from PP were applied to the gel at a final concentration of $20 \mu \mathrm{g}$ of microsomal protein/well. The procedure for Western blot analyses is described in the Methods and Materials section. Three of the 4 duplicate gels were antibody probed and one was protein-stained. Equivalent concentrations of untreated control samples $(\mathrm{CL}=$ control liver; $\mathrm{CPP}=$ control PP $)$ were included on the gels as positive or negative controls.

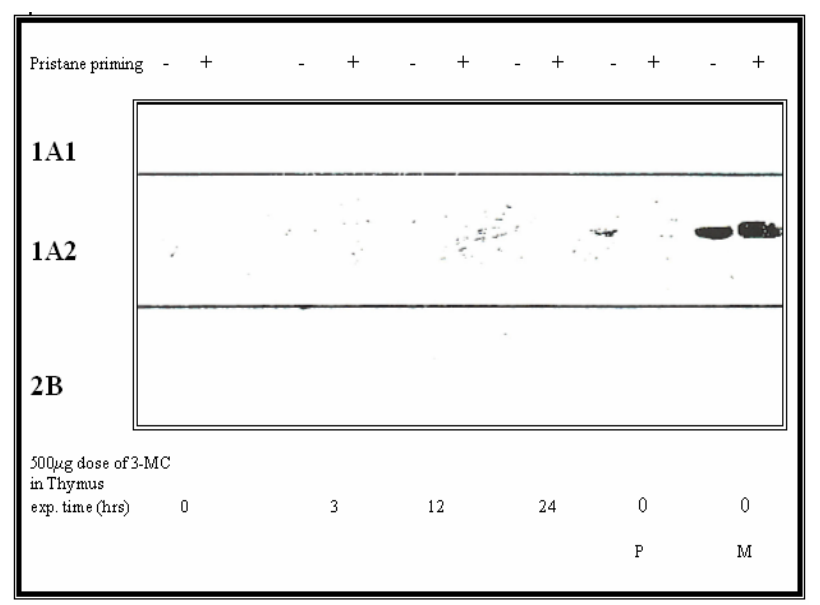

Figure 5: The effects of $500 \mu \mathrm{g} 3-\mathrm{MC}$ injected into the $\mathrm{PP}, 3-\mathrm{MC}$ or $\mathrm{PB}$ very high dose treatment on expression CYP in THY. This immunoblot was generated from thymic microsomal samples from unprimed or pristane primed $(-=$ unprimed; $+=$ pristane primed) rats which were either untreated (CT), carcinogenic dose 3-MC treated $(3,12$ or $24 \mathrm{hr})$, very high dose PB (P) or 3-MC (M) treated. Only thymic microsomal samples were applied to the gel at a final concentration of $200 \mu \mathrm{g}$ of microsomal protein/well. The procedure for Western blot analyses is described in the Methods and Materials section. Three of the 4 duplicate gels were antibody probed and one was Coomassie blue stained.

With respect to the THY (see Fig. 5), the salient points were as follows: First, neither CYP1A1, CYP1A2 nor CYP2B was detected in THY from either normal rats (Group 1), rats treated with only pristane (Group 2) or rats treated with the carcinogenic doses of 3-MC $(0.05,5$ or $500 \mu \mathrm{g}$ ) with (Group 3) or without pristane cotreatment (Group 4). In fact, the only method to elicit detectable amounts of a CYP within THY was by very high dose 3-MC treatment (Group 5), in which case CYP1A2 was the only CYP detected; pristane treatment elicited an increased amount of the CYP1A2 protein. However, very high dose PB treatment (Group 6) did not elicit the expected induction of CYP2B1 or CYP2B2. Furthermore it should be noted that $200 \mu \mathrm{g}$ of microsomal protein was used for the thymic immunoblots (i.e. the maximum protein load for the SDS-PAGE employed), which illustrates differences in the tissue specific expression of the CYP isozymes examined in this study.

\section{Densitometric Scans of Bands on Immunoblots Derived from Western Analyses}

Densitometric scans were employed in these studies for two purposes. One was to assure that equivalent amounts of protein were applied to each gel lane, and the other was to statistically validate any possible differences which were detected on subsequent immunoblots. One representative gel with samples from each different tissue is shown (Fig. 6), but for each group examined, four gels were run, with similar patterns emerging.

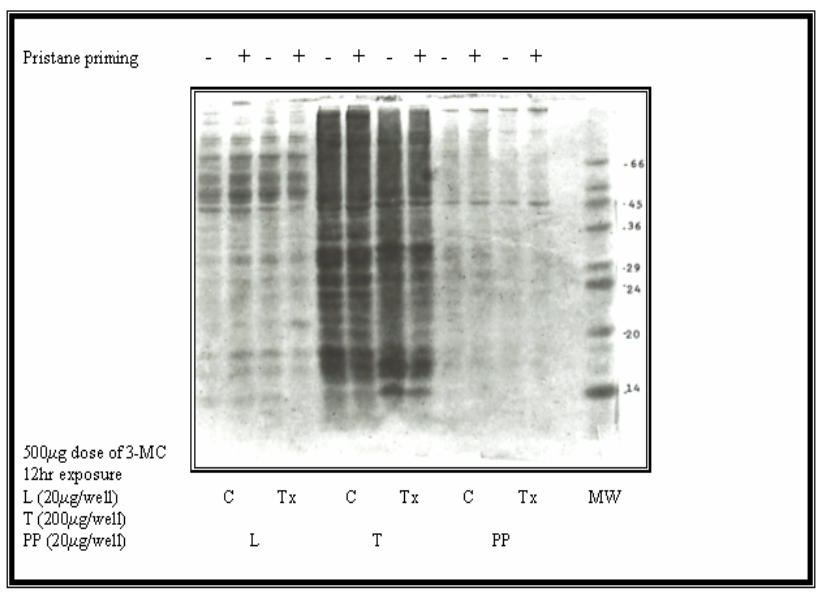

Figure 6: Representative Coomassie blue stained SDSPage gel of rat Peyer's Patches (PP), THY ( $\mathrm{T}=$ thymus) and LIV $(\mathrm{L}=$ liver $)$ microsomal proteins isolated following treatment with $3-\mathrm{MC}(500 \mu \mathrm{g}$ dose; $12 \mathrm{hr}$ exposure) and with $(+=2$ wk pristine priming) or $(-)$ without pristane. Samples were loaded at $20 \mu \mathrm{g}$ of protein/well for PP and L, or $200 \mu \mathrm{g}$ of protein/well for THY samples. Control samples $(\mathrm{C}=$ no $3-\mathrm{MC})$ were run in lanes next to the corresponding $\mathrm{Tx}$ (treatment with $500 \mu \mathrm{g}$ 3-MC directly into the PP) samples for comparison purposes as shown on the immunoblot in Fig. 7. The mobilities of molecular weight markers (MW) are indicated. Gels were electrophoresed according to the Laemmli method.

Based on densitometric scans, variability in the amount of protein present in each gel lane was $\simeq 10 \%$ of the mean value obtained from all respective samples on a particular gel. Regardless of treatment, the scans 
substantiate that the evaluations made in this study represent effects of the chemical treatment and not differences due to non-equivalent amounts of protein within each gel lane or gels. Figures 6 shows a representative gel and Figure 7 represents its subsequent immunoblot.

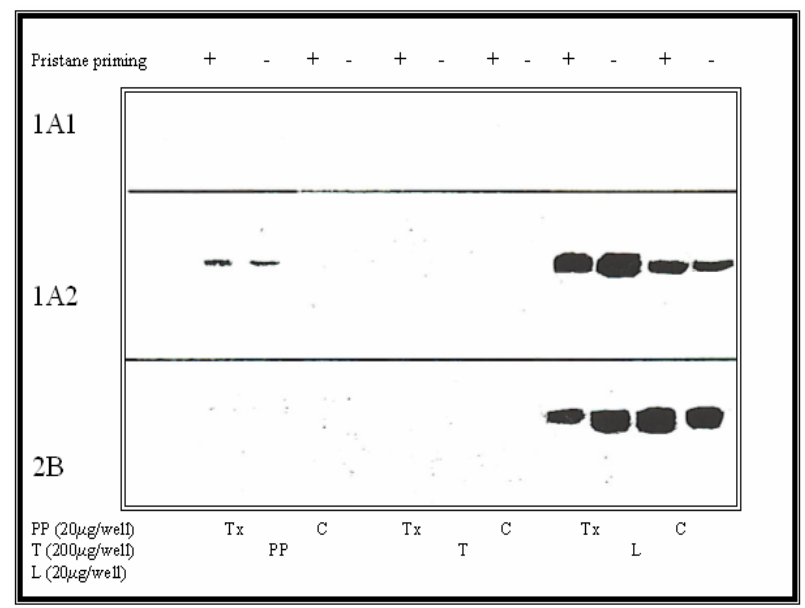

Figure 7: Composite immunoblot of PP, THY (T) and LIV (L) microsomal proteins from unprimed (-) or pristane primed $(+)$ rats which were either untreated $(C)$ or treated with 3-MC (Tx). All tissues were isolated from rats 12 hours after injection of $500 \mu \mathrm{g}$ of $3-\mathrm{MC}$ into the PP. The optimized concentrations of microsomal proteins which were loaded into each of the wells of the gel are noted and the immunoblot generated was probed with MAbs specific for CYP1A1, CYP1A2 or CYP2B as indicated.
These figures illustrate that there were gross differences in the amounts of microsomal material needed in order to conduct these analyses. These differences depended on the tissue, treatment protocol and cytochrome species which was examined. For example, in studies of rats treated with the $500 \mu \mathrm{g}$ carcinogenic dose of 3-MC (Groups $3-4$ ), 20 $\mu \mathrm{g}$ of microsomal protein per gel lane was used for PP and LIV samples and $200 \mu \mathrm{g}$ for the THY samples. With respect to the samples generated using very high doses for either 3-MC (Group 5) or PB (Group 6), only 0.2 $0.5 \mu \mathrm{g}$ of protein was typically needed to detect the CYP within liver samples, as seen in Fig. 2. Another example supporting the need to load different amounts of protein in seen in samples analyzed following PP treatment with the carcinogenic doses of 3-MC (Group 3), only 500 $\mu \mathrm{g}$ of 3-MC elicited detectable levels of CYP1A2 in the PP; in this case pristane co-treatment (Group 4) affected the initial (i.e. $3 \mathrm{hr}$ post 3-MC injection) response, as seen in Fig. 4. With respect to the THY, only very high dose 3MC treatment (Group 5) was effective in inducing CYP and in this case only CYP1A2 was detected; pristane cotreatment led to an augmentation of the response (Fig. 5). Thus, although there may have been differences in the apparent amounts of particular proteins which were present within different tissues or even within a particular tissue isolated after various treatments, the total amount of protein loaded onto each gel lane was equivalent.
Panel A

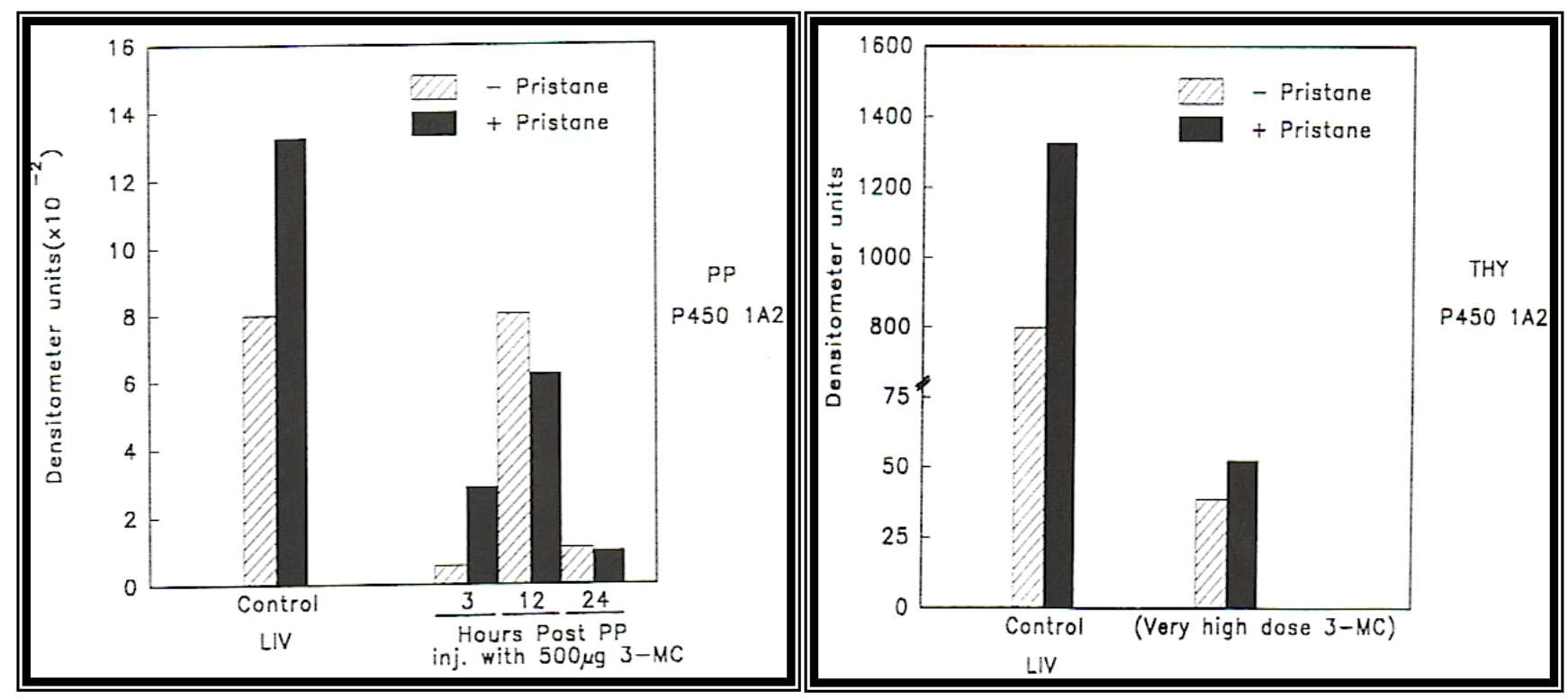

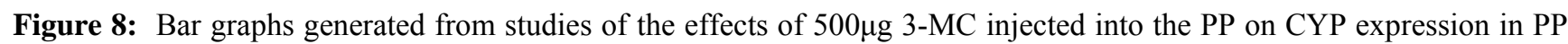
and the effects of very high dose 3-MC treatment on expression of CYP in THY. Densitometric values of bands from immunoblots derived from rat PP (Panel A) and thymic (Panel B) microsomes were graphically represented using SIGMAPLOT. Values were determined by volume integration and represent the sum of all pixel values minus background. Microsomes were prepared from rats following indicated treatment protocols and immunoblots were analyzed as described in the text. The data depict densitometer units versus pristane treatment $(-=$ unprimed; $+=2$ wk pristane primed) or treatment with 3-MC (PP: $3 \mathrm{hr}, 12 \mathrm{hr}$ or 24hr; THY: very high dose, i.p.). Control LIV values are included on each graph as a point of reference. 
Figure 8 represents densitometric compiled values obtained from PP samples following treatment with

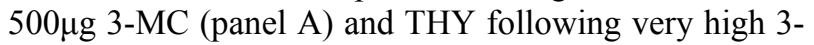
MC dose treatment (panel B) which were adjusted to reflect the amount of microsomal protein used to generate the immunoblot signals. As observed, the levels of CYP1A2 detectable in PP and THY were far below levels detectible in LIV. The data from quantitative analyses of liver samples was tabulated and the averages from four Western blot scans for each condition studied are presented in Table I. The table also summarizes the results of Western analyses of the CYPs examined in this study. This data represent the results of the scans which addressed the effects of basal (Group 1) versus the very high dose (Group 5) or carcinogenic dose (Group 3) effects of 3-MC on CYP in the LIV. In theory any change in intensity of the bands was indicative of a change in the amount of CYP protein expressed as a result of treatment with the indicated compound(s). Therefore, the desitometric scans validated the idea that observed differences were due to different tissues or various treatments, not loading inconsistencies.

Table 1: Composite of Densitometric Values of Immunoblots from Rat Liver Microsomes.

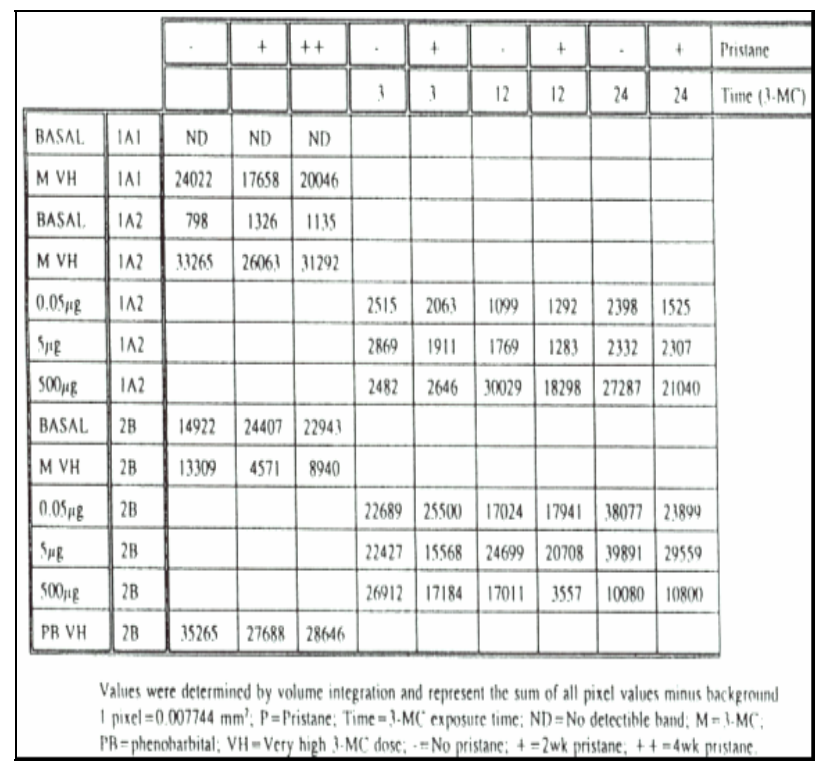

\section{Discussion}

The complexity of chemical carcinogenesis has led to the notion that various regulatory systems respond to exposure to a broad range of endogenous and xenobiotic compounds which can cause cancer. Inherent to this notion is the existence of cellular control mechanisms which adjust to changing biological and biochemical conditions within the cell. Along these lines, the manifestation of cancer appears to be a reflection of modifications to normal cellular events, which involve regulation. One such cellular kinase regulated system involves the CYP monooxygenases, which respond directly to external xenobiotics. With respect to cancer, several lines of evidence support the existence of a relationship between changes in the expression of CYP and carcinogenesis. For example, certain enzymes within the CYP enzyme multigene family have been found to influence the overall process of tumorigenesis and therefore are of interest in studies involving chemical carcinogenesis.

One of the most prevalent classes of chemical carcinogens in the environment which may act as initiators in chemical carcinogenesis is the polycyclic aromatic hydrocarbons (PAH). The PAH employed in this study was 3-MC. Although it has been implicated in many cancers, the parent compound is considered to be chemically inert. However, the 3-MC inducible monooxygenases catalyze the bioactivation of 3-MC eliciting mutagenic, carcinogenic or teratogenic derivatives. The studies presented above examined the possible relationship between basal and/or inducible CYP protein levels and exposure to different doses of 3-MC or pristane under conditions which elicited a preferential induction of different types of lymphoid malignancies.

In the context of target organs specificity, within the PP co-treatment with pristane and low dose 3-MC resulted in only B-cell malignancies and no thymic tumors. On the other hand, with a high dose only thymic tumors developed, and no B cell malignancies were seen [9]. Subsequent studies revealed that within PP CYP1A enzymatic activity observed at low 3-MC dose was no longer detectable following high 3-MC dose treatment [7]. These studies reveal that CYP1A2 protein was

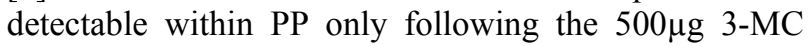
dose treatment and pristane co-treatment was associated with a higher level of CYP1A2, but only at $3 \mathrm{hr}$ after injection of 3-MC. These findings are reproducible and confirm the notion that B-cell tumors occur within PP as a result of CYPIA-catalyzed metabolic activation of low doses of 3-MC, but the abundance of CYP1A2 protein present after high 3-MC dose treatment coupled with the fact that no EROD activity was detectable at this dose treatment [7] infers that CYP1A2 protein was present but inactivated following $500 \mu \mathrm{g}$ dose 3 -MC treatment and that pristane enhances this toxicity and/or carcinogenicity. A possible explanation is that reactive species of 3-MC which can bind to DNA or other macromolecules within the cell poisoned the PP, a gut associated lymphoid organ comprised of predominately B lymphocytes, due to extensive DNA adduct formation in the PP. However, it can not be ruled out that the decreased CYP1A enzymatic activity observed following high dose 3-MC treatment was due to interactions between CYP1A2 and another CYP isozyme.

Comparatively, within THY, the target organ for $\mathrm{T}$ cell malignancies, Western blot analyses revealed that appreciable levels of CYP1A2 protein were expressed only after a very high 3-MC dose treatment. Although THY is not the initial site of injection, there is evidence which supports the fact of dissemination of 3-MC to the THY [9]. Also, the microenvironment within the thymus is predominantly composed of T-cells, as opposed to Bcells within $\mathrm{PP}$, such that reactive species generated from high dose 3-MC treatment may not inactivate the thymus tissue. In light of the observed 3-MC dose effects, the oxidation of 3-MC to reactive species by CYP1A, dissemination of 3-MC to the THY, and in turn, a propensity towards thymic lymphomas following high dose 3-MC treatment, the data presented strongly support 
the hypothesis that very high 3-MC dose treatment affects the induction of thymic lymphomas and are attributable to the levels of CYP1A protein expression.

Target organ specificity is a key determinant in both tumor type and location and pharmacokinetics parameters such as absorption, distribution, dissemination or elimination may be affected by different routes of exposure [16]. Along these lines, the $\mathrm{PP}$ was the injection site in our model. It is possible that expression of the 3-MC inducible CYP isozymes within $\mathrm{PP}$ rendered this tissue more prone to carcinogenesis, whereas the THY was more protected. Within LIV, where most of the CYP isozymes are present, the levels of CYP1A and CYP2B create a balance between toxification and detoxification, thus allowing effective CYP1A induced clearance of 3-MC from LIV and increased susceptibility of target organs. Most associations with chemical carcinogenesis in rats have been CYP1A1 in LIV, but only CYP1A2 was present in detectable levels in our target organs due to our route of exposure. This imbalance between CYP isozymes rendered the PP and THY more suceptible to the deleterious effects of 3-MC and pristane and helps to explain the preferential induction of lymphoid malignancies within these target organs.

In light of the possible mode(s) of action of pristane, membrane perturbation by pristane may alter the phospholipid environment. Along these lines, it has been well established that drug-metabolizing enzymes located in the cell membranes can be affected by compounds which influence membrane structure and fluidity [23]. In other words, the functions of CYP are intimately related and highly dependent upon membrane interactions/function. Previous studies have clearly established that pristane is membrane associated [16] and elicits marked biological effects on membrane fluidity, as well as chromatin conformation [8]. Thus, the premise that pristine perturbs the plasma membrane and leads to altered expression, and/or function of the CYPs, is plausible. This may explain in part how pristane influences a diverse group of pathological processes, including carcinogenesis.

In light of the results presented above and those in a previous study [7], a myriad of effects associated with pristane may have important implications in chemical carcinogenesis. Pristane clearly elicits marked effects on CYP protein expression and enzymatic activity. As such, it may affect cellular susceptibility to carcinogenic metabolites of 3-MC. More importantly, pristane appears to be a factor in the induction of experimental lymphoid malignancies and speculatively may be an etiological dietary factor in human cancer. As such, these data taken in conjunction with other observations from our laboratory, as well as from other investigators, demonstrate the biological relevance of studies to address the mechanism(s) of action of pristane.

Acknowledgments: This work was supported in part by the NIH-EARDA grant, Grant No. 1G11HDO46519-01, from the National Institute of Child Health and Human Development; the Excellence in Partnerships for Community Outreach grant (Grant \# 632301-1210) and through the National Institutes of Health Grant GM 44982.

\section{References}

1. Felix, K.; Potter M.; Bornkamm, G. W.; Janz, S.: In vitro mutagenicity of the plasmacytomagenic agent pristane (2, 6, 10, 14-tetramethylpentadecane). Cancer Lett., 1997, 113(1-2), 71-76.

2. Felix, K.; Gerstmeier, S.; Kelliher, K. A.; Bornkamm, G. W., Janz S. Elevated mutant frequencies in lymphoid tissues persist throughout plasmacytoma development in BALB/c.lambdaLIZ mice. Cancer Res. 1999, 59(15), 3621-3626.

3. Potter, M.; Jones, G.; Dubois, W.; Williams, K.; Mushinski, E.: Myeloma proteins that bind Hsp65 (GroEL) are polyreactive and are found in high incidence in pristine induced plasmacytomas. Curr. Top. Microbiol. Immunol., 2000, 252, 265-271.

4. Felix, K.; Gerstmeier, S.; Kyriakopoulos, A.; Howard, O. M.; Dong, H. F.; Eckhaus, M.; Behne, D.; Bornkamm, G. W., Janz, S.: Selenium deficiency abrogates inflammation-dependent plasma cell tumors in mice. Cancer Res. 2004, 64(8), 2910-2917.

5. Potter, M.; Wax, J. S.; Hansen, C. T.; Kenny, J. J.: BALB/c.CBA/N mice carrying the defective $\mathrm{Btk}^{\mathrm{xid}}$ gene are resistant to pristine-induced plasmacytomagenesis. Internat. Immunol., 1999, 11(7), 1059-1064.

6. Gado, K.; Silva, S.; Paloczi, K.; Domjan, G.; Falus, A.: Mouse plasmacytoma: an experimental model of human multiple myeloma. Haematologica., 2001, 86(3), 227-236.

7. Harper, C. M.; Soni, M. G.; Mehendale, H. M.; Cuchens, M. A.: Pristane-induced effects on cytochrome P-4501A, ornithine decarboxylase and putrescine in rats. Cancer Lett., 1995, 95 (1-2), 11-21.

8. Garrett, L. R.; Cuchens, M. A.: Pristane induced effects on chromatin of rat lymphoid cells. J. Cell. Biochem., 1991, 45, 311-316.

9. Garrett, L. R.; Randall, C. C.; Cuchens, M. A.: Thymic tumors induced by 3-methylcholanthrene treatment of rat Peyer's patches. Exp. Mol. Pathol., 1991, 54, 47-60.

10. Garrett, L. R.; Jones, C. J.; Cuchens, M. A.: Pristane induced gene activation. Chem-Biol Interact., 1981, 119-130.

11. Banbura, M.; Ackland-Berglund, C.; Lee, S.; Hamernik, D.; Jones, C.: Analysis of transcriptional activation of a cyclic AMP response element by 2, 6, 10, 14tetramethylpentadecane (pristane) in JB6 mouse epidermal cells. Mol. Carcino. 1994, 11, 204-214.

12. Garrett, L. R.; Ackland-Berglund, C. E.; Jones, C. J.; Cuchens, M. A.: Differential effects of TPA and pristane on gene expression and transformation in mouse epidermal cells. Exp. Cell. Res. 1993, 205, 416-421.

13. Lee, S. H.; Ackland-Berglund, C. E.; Jones, C. J.: The tumor promoter pristane activates transcription by a cAMP dependent mechanism. Mol. Cell Biochem., 1992, 110, 75-81.

14. Castagna, M.: Phorbol esters as signal transducers and tumor promoters. Biol. Cell, 1987, 59, 3-14.

15. Roesch, S. F.; Wiebel, F. J.: Differential effects of 12-O-tetradecanolyphorbol 13-acetate on cytochrome P-450I and suppression of P-450II. Toxicol., 1990, 61, 147-159. 
16. Bost, K. L.; Cuchens, M.: The role of Peyer's patches in carcinogenesis. I. The adsorption from the gut and retention of 3-methylcholanthrene by Peyer's patches. Carcinogeno. 1986, 7, 1251-1256.

17. Malins, D. C.; Anderson, K. M.; Gilman, N. K.; Green, V. M.; Barker, E. A.; Hellstrom, K. E.: Development of a cancer DNA phenotype prior to tumor formation. Proc. Natl. Acad. Sci. U SA, 2004, 101(29), 10721-10725.

18. Fujii-Kuriyama, Y.; Imataka, H.; Sogawa, K.; Yasumoto K.; Kikuchi, Y.: Regulation of CYP1A1 expression. FASEB J., 1992, 6, 706-710.

19. Sesardic, D.; Cole, K. J.; Edwards, R. J.; Davies, D. S.; Thomas, P. E.; Levin, W.; Boobis, A. R.: The inducibility and catalytic activity of cytochromes P450s (P450IA1) and P450d (P450IA2) in rat tissues. Biochem. Pharmacol., 1990, 39, 499-506.

20. Zacharova, L.Y.; Gulyaeva, L. F.; Lyakhovich, V. V.; Mikhailova, O. N.; Timofeeva, O. A.; Filipenko, M. L.; Kaledin, V. I.: Cytochrome P4501A1 and 1A2 gene expression in the liver of 3methylcholanthrene- and o-aminoazotoluene-treated mice: a comparison between PAH-responsive and PAH-nonresponsive strains. Toxicol. Sci., 2003, 73(1), 108-113.

21. Wang, J. S.; Backman, J. T.; Taavitsainen, P.; Neuvonen, P. J.; Kivisto, K. T.: Involvement of CYP1A2 and CYP3A4 in lidocaine N-deethylation and 3-hydroxylation in humans. Drug Metab. Dispos., 2000, 28(8), 959-965.

22. Yamazaki, H.; Inoue, K., Shaw, P. M.; Checovich, W. J.; Guengerich, F. P.; Shamada, T.: Different contributions of cytochrome P450 2C19 and 3A4 in the oxidation of omeprazole by human liver microsomes: effects of contents of these two forms in individual human samples. J. Pharmacol. Exp. Ther. 1997, 283(2), 434-442.

23. Yoo, J. S.; Smith, T. J.; Ning, S. M.: Modulation of the levels of cytochromes $\mathrm{P} 450$ in rat liver and lung by dietary lipid. Biochem. Pharmaco., 1992, 43, 2535-2542.

24. Miller, M. S.; Jones, A. B.; Chauhan, D. P.; Park, S. S.; Anderson, L. M.: Induction of cytochrome P450IA1 in fetal rat liver by a single dose of 3methylcholanthrene. Biochem. Biophys. Res. Comm., 1991, 176, 280-287.

25. Peng, J. Z.; Remmel, R. P.; Sawchuk, R. J.: Inhibition of murine cytochrome P4501A by tacrine: in vitro studies. Drug Metab. Dispos,, 2004, 32(8), 805-812.

26. Shimada, T.; Sugie, A.; Yamada T.; Kawazoe H.; Hashimoto, M.; Azuma, E.; Nakajima, T.; Inoue, K.; Oda, Y.: Dose-response studies on the induction of liver cytochromes P4501A1 and 1B1 by polycyclic aromatic hydrocarbons in arylhydrocarbonresponsive C57BL/6J mice. Xenobiotica., 2003, 33(9), 957-971.

27. Perloff, M. D.; von Moltke, L. L.; Greenblatt, D. J.: Ritonavir and dexamethasone induce expression of CYP3A and P-glycoprotein in rats. Xenobiotica, 2004, 34(2), 133-150.

28. Robottom-Ferreira, A. B.; Aquino, S. R.; Queiroga, R.; Albano, R. M.; Ribeiro Pinto, L. F.: Expression of CYP2A3 mRNA and its regulation by 3methylcholanthrene, pyrazole, and B-ionone in rat tissues. Braz. J. Med. Biol. Res,, 2003, 36(7), 839-844.

29. Yang, C. S.; Patten, C. J.; Ishizaki, H.; Yoo, J. S. H.: Induction, purification and characterization of cytochrome P450 IIE. Methods in Enzymol., 1990, 206, 595-601.

30. Sueyoshi, T.; Negishi, M.: Phenobarbital response of cytochrome P450 genes and nuclear receptors. Annual Rev. of Pharmacol. and Toxicol. 2001, 41, 123-143.

31. Thomas, P. E.; Reik, L. M.; Ryan, D. E.; Levin, W.: Characterization of nine monoclonal antibodies against rat hepatic cytochrome P450c. Delineation of at least five spatially distinct epitopes. J. Biol. Chem., 1994, 259, 3890-3899.

32. Reik, L. M.; Maines, S. L.; Ryan, D. E.; Levin, W.; Bandiera, S. B.; Thomas, P. E.: A simple, nonchromatographic purification procedure for monoclonal antibodies. Isolation of monoclonal antibodies against cytochrome P450 isozymes. $J$. Immunol. Methods, 1987, 100, 123-130.

33. Dunning, W. F.; Curtis, M. R.: The respective roles of longevity and genetic specificity in the occurrence of spontaneous tumors in hybrids between two inbred lines of rats. Cancer Res., 1946, 6, 61-68.

34. Howard, C. B.; Stevens, J.; Izevbigie, E. B.; Walker, A.; McDaniel, O.: Time and dose-dependent modulation of phase 1 and phase 2 gene expression in response to treatment of $\mathrm{MCF}-7$ cells with a natural anti-cancer agent. Cell. Mol. Biol., 2003, 49, 1057-1065. 